\title{
Enhancement of Ultrasound Images (Removing of Speckle Noise Using Special An Isotropic Diffusion Filter)

\author{
Prof.(Dr.) Hitesh H Vandra
} \\ Shree Swaminarayan Institute of Technology, Bhat, Gujarat, India
}

\section{ABSTRACT}

Medical imaging techniques are predominantly used in medical diagnosis and therapy. The success of this technique depends largely on the quality of images. Due to various factors images do not have appropriate contrast and are often overridden by noise, making the interpretation of the images too difficult leading to incorrect diagnosis. This will be a very important and significant contribution to the medical professional. Removing noise from the original image is still a challenging research in image processing. Generally there is no common enhancement approach for noise reduction. Several approaches have been introduced and each has its own assumption, advantages and disadvantages. The speckle noise is commonly found in the ultrasound medical images. This paper presents different filtering techniques based on Statistical methods for the removal of speckle noise in ultrasound images. The quality of the enhanced images is measured by the Statistical quantity measures: Peak Signal-to Noise Ratio (PSNR), Mean Square Error (MSE) and Correlation coefficient (COC).

Keywords : Ultrasound Images, Speckle Noise, Diffusion filter, speckle reduction anisotropic diffusion filter, PSNR, MSE, COC $(\beta)$

\section{INTRODUCTION}

Ultrasound imaging is a widely used and safe medical diagnostic technique, due to its noninvasive nature, low cost and capability of forming real time imaging. However the usefulness of ultrasound imaging is degraded by the presence of signal dependant noise known as speckle. The speckle pattern depends on the structure of the image tissue and various imaging parameters. There are two main purposes for speckle reduction in medical ultrasound imaging (1) to improve the human interpretation of ultrasound images (2) despeckling is the reprocessing step for many ultrasound image processing tasks such as segmentation and registration. A number of methods have been proposed for speckle reduction in ultrasound imaging. While incorporating speckle reduction techniques as an aid for visual diagnosis, it has to keep in mind that certain speckle contains diagnostic information and should be retained. The objective of this paper is to give an overview about types of speckle reduction techniques in ultrasound imaging.

ULTRASOUND imaging application in medicine and other fields is enormous. It has several advantages over other medical imaging modalities. 


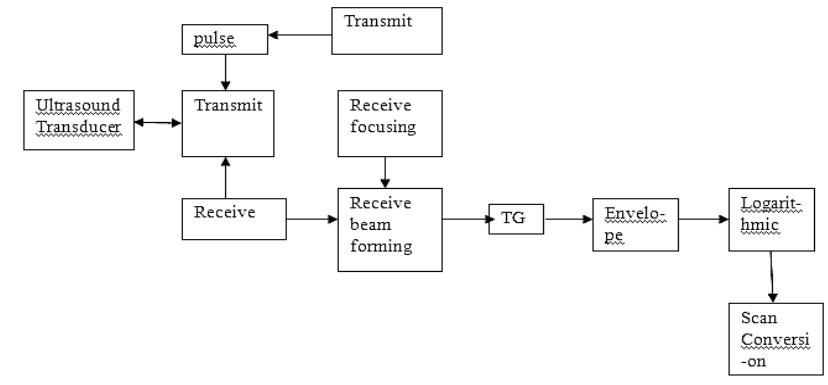

Figure 1. Block diagram of ultrasound imaging system

The use of ultrasound in diagnosis is well established because of its noninvasive nature, low cost, capability of forming real time imaging and continuing improvement in image quality. It is estimated that one out of every four medical diagnostic image studies in the world involves ultrasonic techniques. US waves are characterized by frequency above $20 \mathrm{KHz}$ which is the upper limit of human hearing. In medical US applications, frequencies are used between $500 \mathrm{KHz}$ and $30 \mathrm{MHz}$ B-mode imaging is the most used modality in medical US. An US transducer which is placed onto the patient's skin over the imaged region sends an US pulse which travels along a beam into the tissue. Due to interfaces some of the US energy is reflected back to the transducer which converts it into echo signals. These signals are then sent into amplifiers and signal processing circuits in the imaging machine's hardware to form a 2-D image. This process of sending pulses launched in different directions is repeated in order to examine the whole region in the body. Thus, US imaging involves signals which are obtained by coherent summation of echo signals from scatterers in the tissue.

In many cases volume quantification is important in assessing the progression of diseases and tracking progression of response to treatment. Thus, 3D ultrasound imaging has drawn great attention in recent years.

\section{SPECKLE NOISE}

Speckle noise is the characteristic seen in ultrasound images that contribute to the visual noise. The image of a relatively uniform object with many scattering sources within a resolution cell will have pixel values that vary randomly with position due to constructive and destructive interference.

Speckle is not a noise in an image but noise-like variation in contrast. It arises from random variations in the strength of the backscattered waves from objects and is seen mostly in medical imaging.

\section{NEED FOR SPECKLE REDUCTION}

Thus, speckle is considered as the dominant source of noise in ultrasound imaging and should be processed without affecting important image features. The main purposes for speckle reduction in medical ultrasound imaging are:

To improve the human interpretation of ultrasound images - speckle reduction makes an ultrasound image cleaner with clearer boundaries.

Despeckling is a preprocess step for many ultrasound image processing tasks such as segmentation and registration - speckle reduction improves the speed and accuracy of automatic and semiautomatic segmentation \& registration.

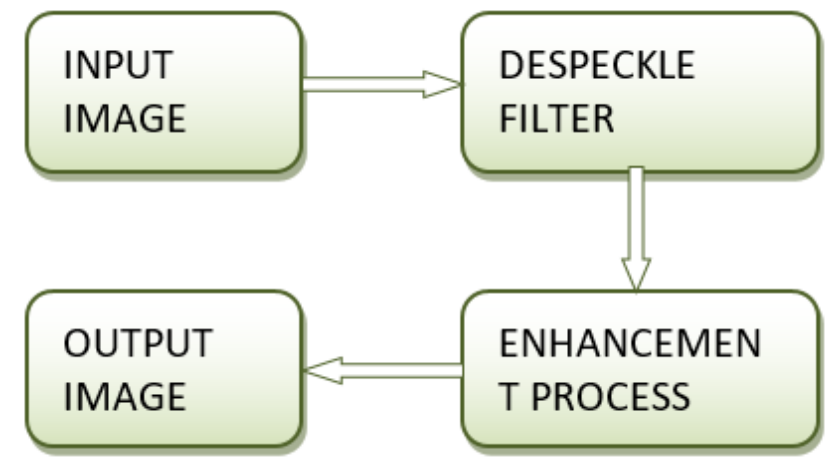

Figure 2. Block diagram of Enhancement of ultrasound imaging system 


\section{Diffusion Filtering}

Diffusion filters remove the noise from an image by modifying the image via solving a partial differential equation (PDE). Smoothing is carried out depending on the image edges and their directions. Anisotropic diffusion is an efficient nonlinear technique for simultaneously performing contrast enhancement and noise reduction. It smoothes homogeneous image regions, but retains image edges without requiring any information from the image power spectrum. It may, thus, directly be applied to images. Consider applying the isotropic diffusion equation given by $\frac{d g_{i, j, t}}{d t}=\operatorname{div}(d \nabla g)$ using the original noisy image $g_{i, j, t=0}$ as the initial condition, where $g_{i, j, t=0}$ is an image in the continuous domain, $i$ and $j$ specify the spatial position, $t$ is an artificial time parameter, $d$ is the diffusion constant, and $\nabla g$ is the image gradient. Modifying the image according to this linear isotropic diffusion equation is equivalent to filtering the image with a Gaussian filter. In this paper, we will present conventional anisotropic diffusion, speckle-reducing anisotropic diffusion.

\section{Anisotropic Diffusion Filtering (DsFad)}

Perona and Malik replaced the classical isotropic diffusion equation, as described above, by the introduction of a function $d i, j, t=f(|\nabla g|)$ that smoothes the original image while trying to preserve brightness discontinuities with

$$
\frac{d g_{i, j t}}{d t}=\operatorname{div}\left[d_{i, j, t} \nabla g_{i, j, t}\right]=\left[\frac{d}{d_{i}} d_{i j, t} \frac{d}{d_{i}} g_{i j, t}\right]+\left[\frac{d}{d_{j}} d_{i j, t} \frac{d}{d_{j}} g_{i j, t}\right]
$$

$$
\text { ....1.a }
$$

Where $|\nabla g|$ is the gradient magnitude, and $d(|\nabla g|)$ is an edge stopping function, which is chosen to satisfy $d \rightarrow 0$ when $|\nabla g| \rightarrow \infty$, so that the diffusion is stopped across edges. This function, called the diffusion coefficient $d(|\nabla g|)$, which is a monotonically decreasing function of the gradient magnitude $|\nabla g|$, yields intraregion smoothing and not inter region smoothing by impeding the diffusion at image edges. It increases smoothing parallel to the edge and stops smoothing perpendicular to the edge, as the highest gradient values are perpendicular to the edge and dilated across edges. The choice of $d(|\nabla g|)$ can greatly affect the extent to which discontinuities are preserved. For example, if $d(|\nabla g|)$ is constant at all locations, then smoothing progresses in an isotropic manner. If $d(|\nabla g|)$ is allowed to vary according to the local image gradient, then we have anisotropic diffusion. A basic anisotropic PDE is given in Eq. (1.a).

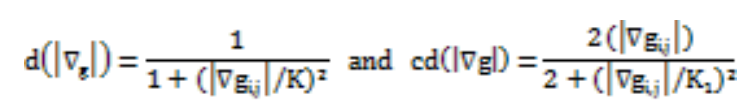

where $K$ and $K 1$ are positive gradient threshold parameters, known as diffusion or flow constants. In this book, the first diffusion coefficient in Eq. (1.b) was used as it was found to perform better in our images.

A discrete formulation of the anisotropic diffusion in Eq. (1.a) is

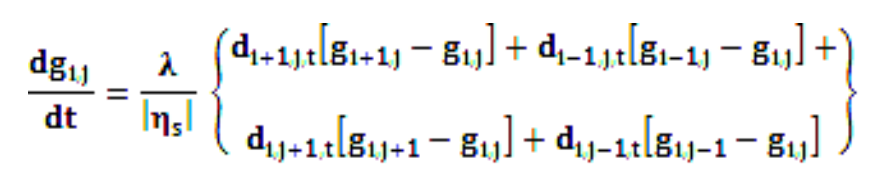

Where the new pixel gray value fi,j at location $i, j$ is

$$
\mathbf{f}_{1, j}=g_{1, j}+\frac{1}{4} \frac{\mathbf{g g}_{1, j}}{d t}
$$

where $d_{i+1, j, t}, d_{i-1, j, t_{2}} d_{i, j+1, t}$, and $d_{i, j-1, t}$ are the diffusion coefficients for the west, east, north, and south pixel directions, respectively, in a fourpixel neighborhood around the pixel $i, j$ where diffusion is computed. The diffusion coefficient leads to the largest diffusion where the nearest-neighbor difference is largest (the largest edge), whereas the smallest diffusion is calculated where the nearest neighbor difference is smallest (the weakest edge). The constant $\lambda \in R^{+}$is a scalar that determines the rate of diffusion, $\eta_{s}$ represents the spatial 
neighborhood of pixel $i, j$, and $\left|\eta_{s}\right|$ is the number of neighbors (usually four except at the image boundaries). Perona and Malik linearly approximated the directional derivative in a particular direction as $\nabla g_{i, j}=g_{i+1, j}-g_{i, j}$ (for the east direction of the central pixel $i, j$ ). Modifying the image according to Eq. (2), which is a linear isotropic diffusion equation, is equivalent to filtering the image with a Gaussian filter. The parameters for the anisotropic diffusion filter used in this $\lambda=0.25, \eta_{s}=8$, and $K=30$, which was used for the calculation of the edge stopping function $d(|\nabla g|)$, in Eq. (1.b).

\section{Speckle-Reducing Anisotropic Diffusion Filtering}

The essence of speckle-reducing anisotropic diffusion is the replacement of the gradient-based edge detector $c d(|\nabla g|)$ in an original anisotropic diffusion PDE with the instantaneous coefficient of variation that is suitable for speckle filtering $\operatorname{csrad}(\mid$ $\nabla g \mid)$. The speckle-reducing anisotropic diffusion filter uses two seemingly different methods, namely, the Lee and Frost diffusion filters. A more general updated function for the output image by extending the PDE versions of the despeckle filter is

$$
f_{i, j}=g_{i_{j}, j}+\frac{1}{\eta_{s}} \operatorname{div}\left(c_{s r a d}(|\nabla g|) \nabla g_{i_{i, j}}\right) \quad \ldots 3
$$

The diffusion coefficient for the speckle anisotropic diffusion $c \operatorname{csad}(|\nabla g|)$ is derived [5] as

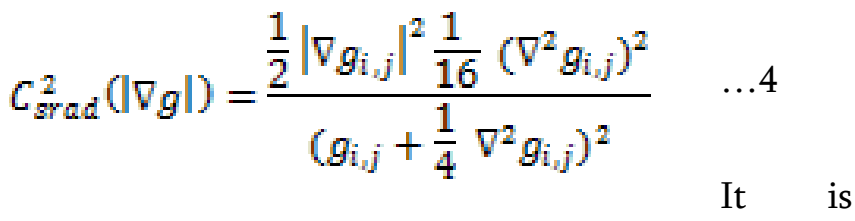

required that $\operatorname{csrad}(|\nabla g|) \geq 0$. The above instantaneous coefficient of variation combines a normalized gradient magnitude operator and a normalized Laplacian operator to act like an edge detector for speckle images. A high relative gradient magnitude and a low relative Laplacian indicate an edge. The DsFsrad filter utilizes speckle-reducing anisotropic diffusion after Eq. (2.b) with the diffusion coefficient $c \operatorname{srad}(|\nabla g|)$ in Eq. (4).

\section{PROPOSED ALGORITHM FOR DESPECKLE FILTER}

\section{STEP-1 Load the image for filtering.}

STEP-2 Specify the original image to be filtered $(K)$, the number of iterations $(n)$, the time step (lambda), and the region of interest to be filtered (rect).

STEP-3 Transform the original image to double and normalize it to $f i, j=(f i, j-\min$ pixel value $) /(\max$ pixel value - min pixel value), where min pixel value and max pixel value represent the minimum and maximum pixel values in the image.

STEP-4 Starting from the left upper corner of the image, select a [3*3] pixel neighbourhood and compute a new grayscale value according to Eq.

$f_{i, j}=g_{i, j}+\frac{1}{\eta_{s}} \operatorname{div}\left(c_{a r a d}(|\nabla g|) \nabla g_{i_{i, j}}\right)$

STEP-5 Assign the new grayscale value to the middle pixel in each window.

STEP-6 Repeat steps 4 and 5 for the whole image by sliding the moving window from left to right.

STEP-7 Repeat steps 4-6 for niter iterations.

STEP-8 Compute the image quality measurement parameter for the original and de-speckled images.

STEP-9 Display the original and despeckled images, the image quality measurement parameters.

\section{EXPERIMENTAL ANALYSIS AND DISCUSSION}

The proposed algorithms have been implemented using image processing tool box of MATLAB 7.0. The performance of various spatial enhancement approaches are analyzed and discussed. The measurement of image enhancement is difficult to measure. There is no common algorithm for the enhancement of the image. The statistical measurement could be used to measure enhancement of the image. The Mean Square Error (MSE), Peak Signal-to-Noise Ratio (PSNR) and correlation coefficient $(\beta)$ are used to evaluate the enhancement performance. The noise level is measured by the standard deviation of the image:

$\sigma=\sqrt{\frac{1}{N}} \sum(b i-b)^{2}$ 
Where, $\mathrm{i}=1,2,3, \ldots, \mathrm{N}, \mathrm{b}$ is the mean gray level value of the original image and bi is the gray level value of the surrounding region and $\mathrm{N}$ is the total number of pixel in the image.

TABLE I. QUALITY MEASUREMENT PARAMETER

\begin{tabular}{|l|l|}
\hline $\begin{array}{l}\text { Statistical } \\
\text { Measurement }\end{array}$ & Formula \\
\hline MSE & $\frac{\left.\sum f(i, j)-F(i, j)\right)^{2}}{M N}$ \\
\hline PSNR & $20 \log _{10} \frac{255}{\text { RMSE }}$ \\
\hline $\begin{array}{l}\text { CORRELATION } \\
\text { COEFFICIANT } \\
(\beta)\end{array}$ & $\frac{\sum(\mathrm{g}-\overline{\mathrm{g}})(\mathrm{g}-\overline{\mathrm{g}})}{\sqrt{\sum(\mathrm{g}-\overline{\mathrm{g}})^{2} \sum(\hat{\mathrm{g}}-\overline{\mathrm{g}})^{2}}}$ \\
\hline
\end{tabular}

The parameters which are used in the filter performance appraisal are Mean Square Error (MSE), Peak Signal to Noise Ratio (PSNR). Where, $\mathrm{f}$ is the original image $\mathrm{F}$ is the filtered image and $\mathrm{MN}$ is the size of image. Where, $g$ and $\grave{U} g$ are original and denoised images respectively and $g$ and $\mathrm{U} g$ are the mean's of the original image and de-noised image respectively.
MSE: is an estimator in many ways to quantify the amount by which an filtered/noisy image differs from noiseless image.

PSNR: is the ratio between possible power of a signal and the power of corrupting noise that affects the fidelity of its representation.

CORRELATION COEFFICIENT: Correlation indicates the strength and direction of linear relationship between two signals and its value lie between +1 to -1 . The original ultrasound image and filtered images of the focal liver lesions obtained by various filtering techniques are shown in figure. If the value of MSE is low and the values of $\beta$ is low and PSNR are larger than the enhancement approach is better.

The performance analysis of the proposed approaches and existing approaches with the regard to ultrasound medical images for focal liver lesions.

- After getting enhanced image, I have concerned Radiology doctor.

- As par doctor opinion this enhanced image is very Impressive and informative.

It should be more useful for radiologists to interpret images and arrive at a perfect diagnosis.

\section{EXPERIMENTAL RESULTS}

Result of De-speckle filter

Original De-speckle

Figure 3: Focal lever lesions
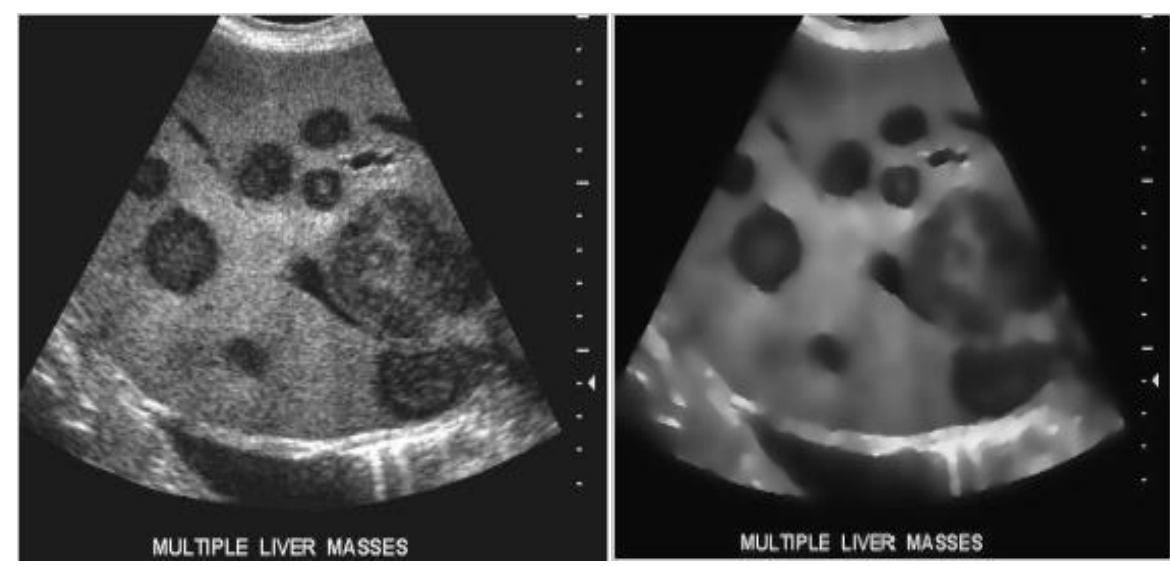
Figure 4: Prostate cancer cell
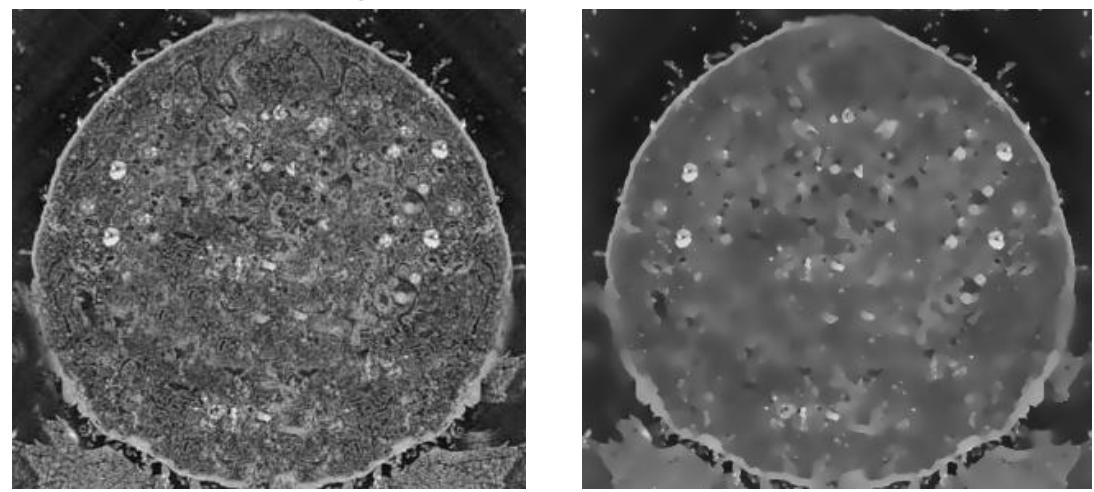

Figure 5: Focal Hepatic Lesions
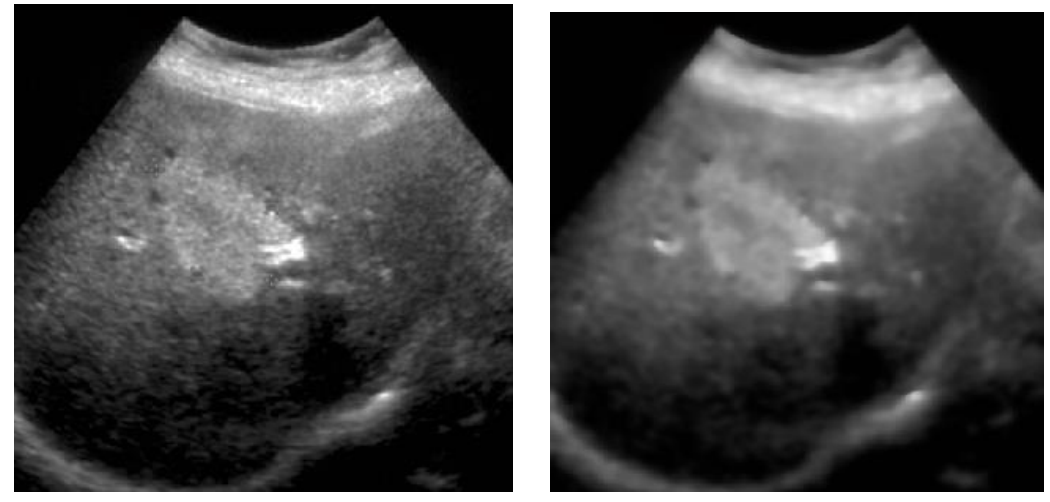

Figure 6: Neurofibroma
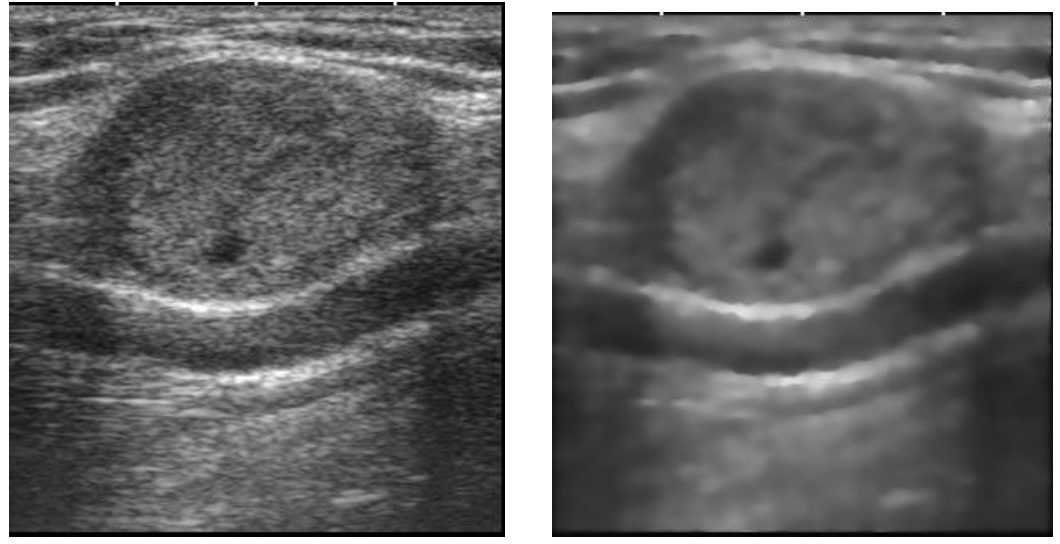

Figure 7: Pancreas
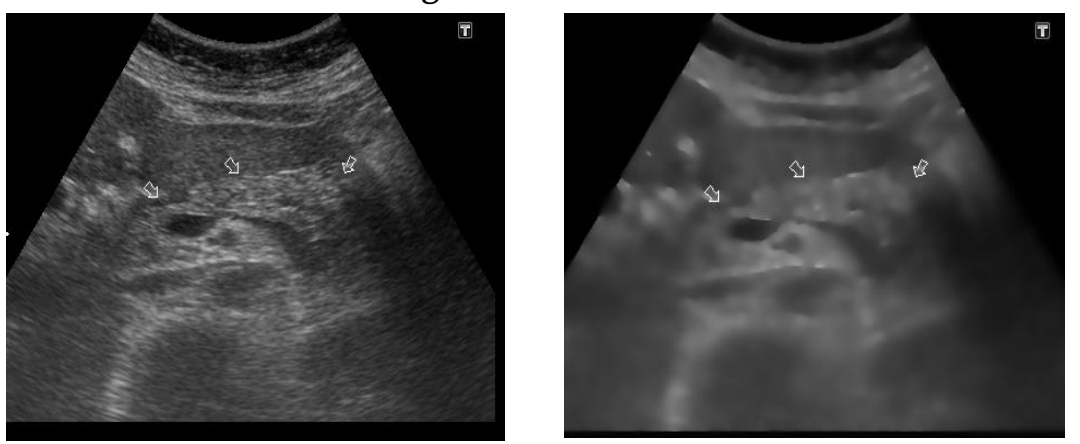

Figure 8: Kidney 

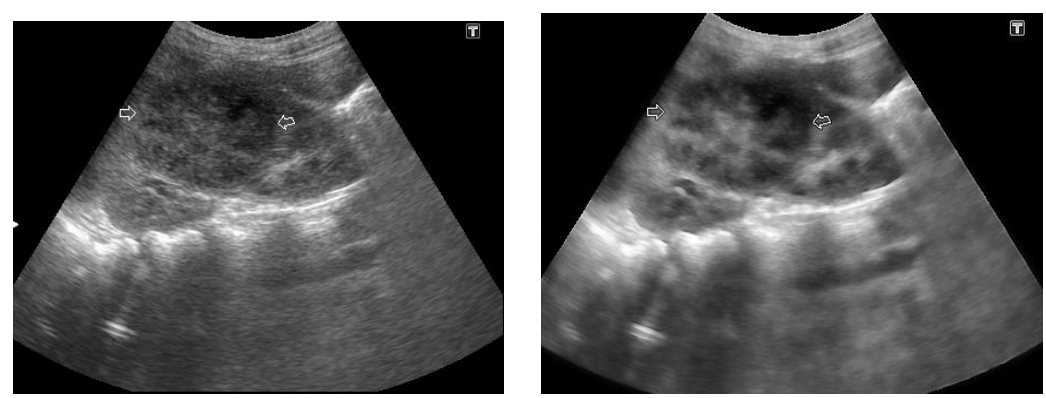

Figure 9: Abdomen
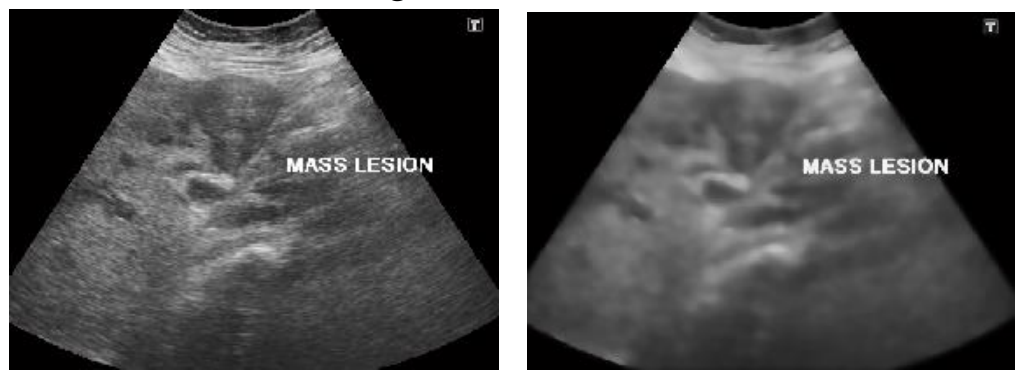

TABLE II. QUALITY MEASUREMENT PARAMETER

\begin{tabular}{|c|c|c|c|c|}
\hline $\begin{array}{c}\text { Sr.n } \\
\text { o }\end{array}$ & Ultrasound Images & MSE & PSNR & COC \\
\hline 1 & focal liver lesions & 0.0024 & 74.27 & 0.96 \\
\hline 2 & Prostate cancer cell & 0.0047 & 71.43 & 0.98 \\
\hline 3 & Focal Hepatic Lesions & 6.41 & 80.05 & 0.99 \\
\hline 4 & Neurofibroma & 0.0014 & 76.68 & 0.98 \\
\hline 5 & Pancreas & 9.12 & 78.53 & 0.99 \\
\hline 6 & Kidney & 9.66 & 78.28 & 0.99 \\
\hline 7 & Abdomen & 0.0013 & 76.97 & 0.98 \\
\hline
\end{tabular}

Result of Enhancement Process

Original Enhanced

Figure 10: Focal lever lesions
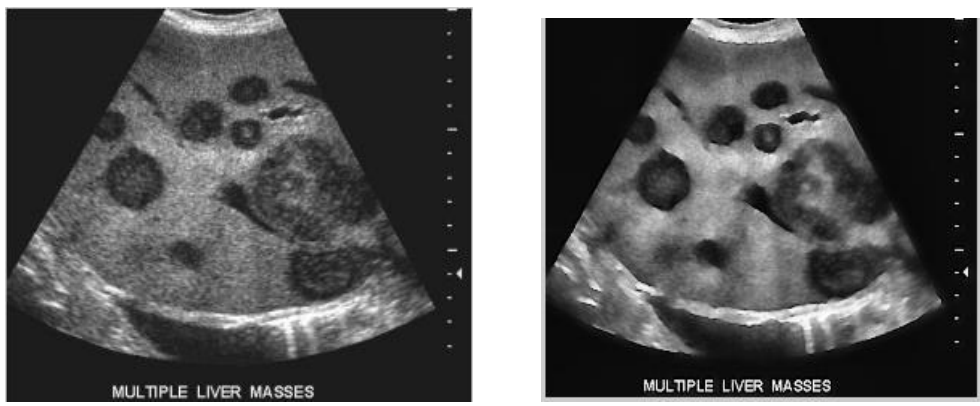

Figure 11: Prostate cancer cell 

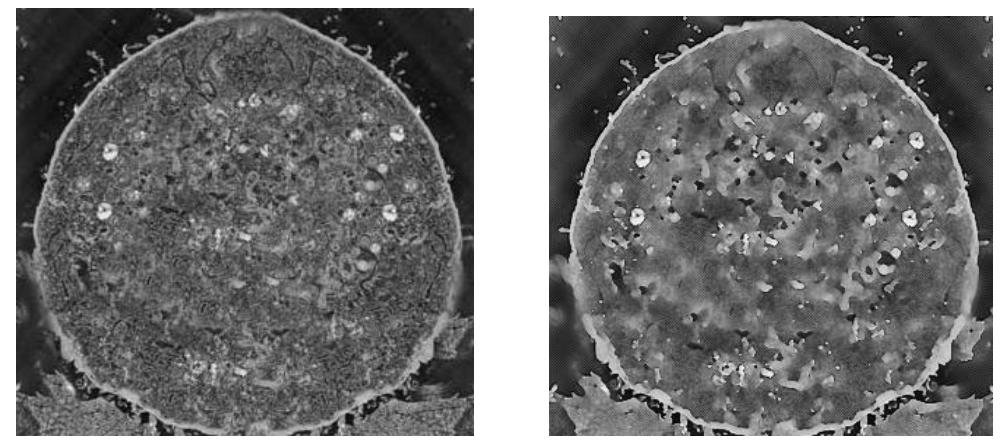

Figure 12: Focal Hepatic Lesions
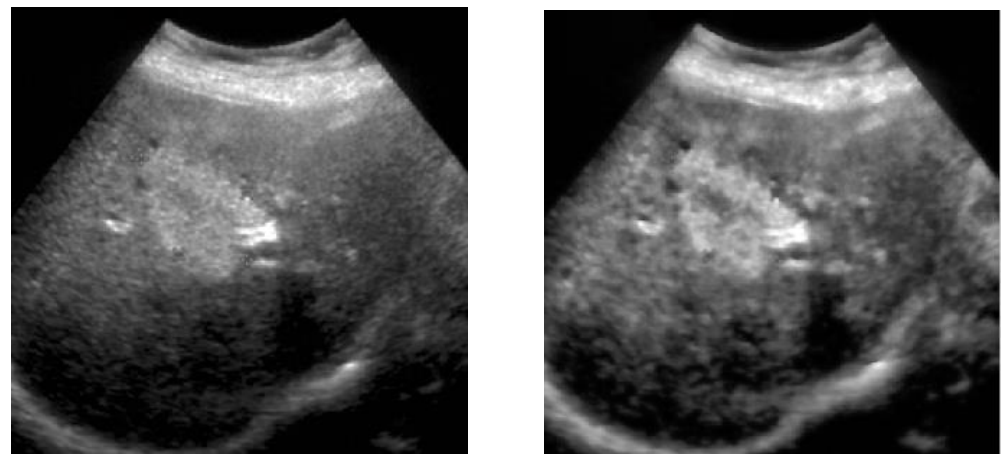

Figure 13: Neurofibroma
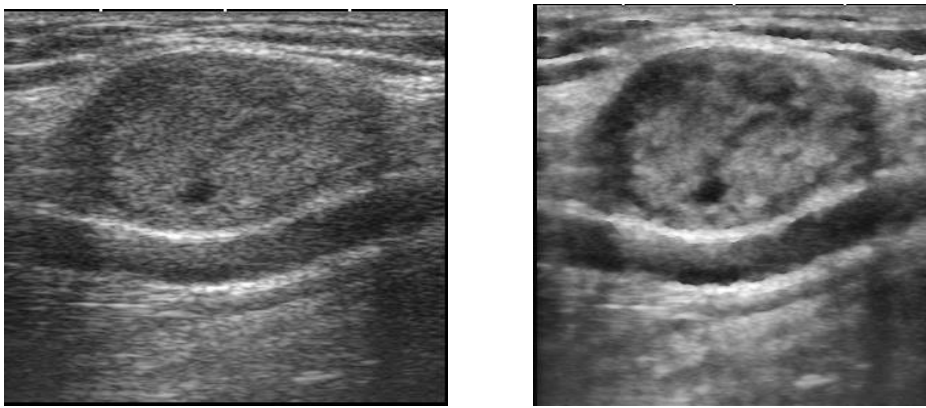

Figure 14: Pancreas
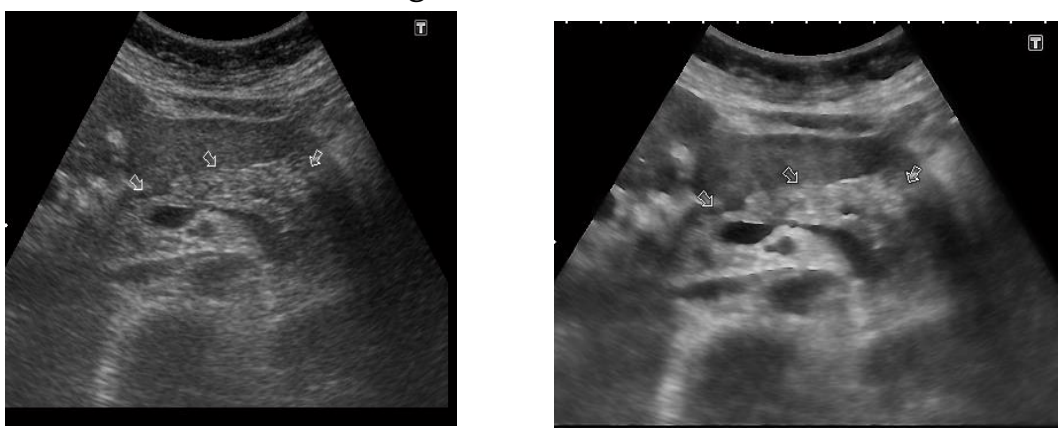

Figure 15: Kidney
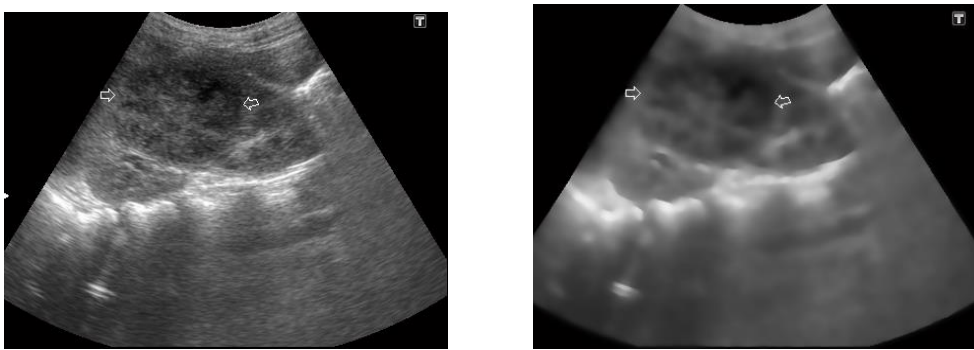

Figure 16: Abdomen 

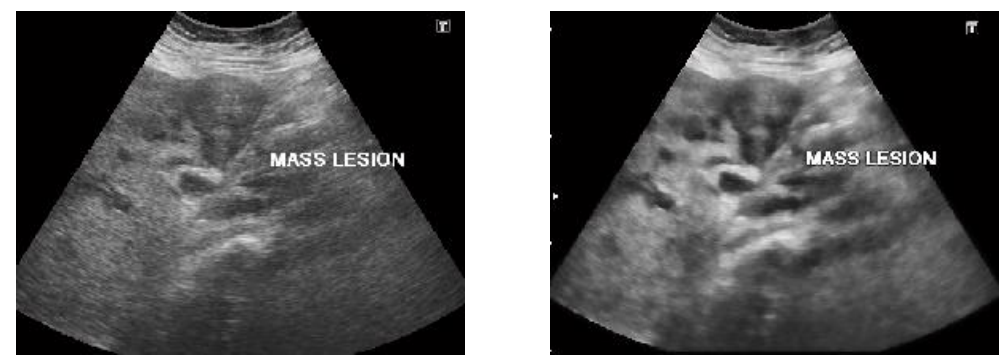

TABLE III. COMPUTATIONAL RESULTS OF ENHANCED IMAGES

\begin{tabular}{|c|c|c|c|c|}
\hline Sr.no & Ultrasound Images & MSE & PSNR & COC \\
\hline 1 & focal liver lesions & 0.0043 & 71.79 & 0.99 \\
\hline 2 & Prostate cancer cell & 0.0076 & 69.33 & 0.98 \\
\hline 3 & $\begin{array}{c}\text { Focal Hepatic } \\
\text { Lesions }\end{array}$ & 0.0054 & 70.82 & 0.98 \\
\hline 4 & Neurofibroma & 0.0022 & 74.75 & 0.99 \\
\hline 5 & Pancreas & 0.0028 & 73.64 & 0.98 \\
\hline 7 & Kidney & 0.0016 & 76.07 & 0.99 \\
\hline 8 & Abdomen & 0.0019 & 75.45 & 0.99 \\
\hline
\end{tabular}

The net result is an image in which small details were enhanced and the background tonality was perfectly preserved. Results like these have made Laplacian based enhancement a fundamental tool used frequently for sharpening Digital images.

\section{CONCLUSION}

The performance of noise removing filtering techniques is measured using quantitative performance measures such as PSNR and MSE as well as in term of visual quality of the images. Many of the methods fail to remove speckle noise present in the ultrasound medical image, since the information about the variance of the noise may not be identified by the methods. The computational result showed one of the proposed filtering techniques.

\section{REFERENCES}

[1]. Rafael Gonzalez, Richard E. Woods, "Digital Image Processing" Second Edition, Pears on Education, 2002.

[2]. Rafael Gonzalez, Richard E. Woods, "Digital Image Processing using MATLAB" low price Edition, Pears on Education, 2004.

[3]. Jain AK, "Fundamentals of Digital Image Processing" Prentice Hall, 1989.

[4]. Atam P.Dhavan, H.K.Haung, Dae-Shik Kim "principles and advance methods in medical imaging and image analysis"

[5]. J. G. Abbott and F. L. Thurstone, "Acoustic speckle:Theory and experimental analysis," Ultrason. Imag., vol. 1, pp. 303-324, 1979.

[6]. Image Processing Fundamentals - Statistics, "Signal to Noise Ratio", 2001.

[7]. S.Kalaivani Narayanan and R.S.D.Wahidabanu: "International Journal of 
Signal Processing, Image Processing and Pattern Recognition Vol. 2, No.3, September 2009"

[8]. L. H. Xie, Pierce, and F. T. Ulaby, "Sar speckle reduction using wavelet denoising mand markov random _eld modeling," IEEE Trans. Geosci. Remote Sensing, vol. 40, no. 10, Oct. 2002."

[9]. Z. Shi and K. B. Fung, "A comparison of digital speckle Filters," in Proc. IEEE on IGARSS ('94), vol. 4, Aug. 1994,

[10]. J. Canny, "A computational approach to edge detection," IEEE Trans.Pattern Anal. Machine Intell., vol. PAMI-8, 1986.

[11]. V. S. Frost, J. A. Stiles, K. S. Shanmugan, and J. C. Holtzman, "Amodel for radar images and its application to adaptive digital filteringof multiplicative noise," IEEE Trans. Pattern Anal. Machine Intell., vol.PAMI-4, pp. 157165, 1982

[12]. J. S. Jin, Y. Wang, and J. Hiller, "An adaptive nonlinear diffusion algorithm for filtering medical images," IEEE Trans. Inform. Technol. Biomed., vol. 4, pp. 298-305, Dec. 2000.

[13]. D. T. Kuan, A. A. Sawchuk, T. C. Strand, and P. Chavel, "Adaptive restoration of images with speckle," IEEE Trans. Acoust., Speech, Signal Processing, vol. ASSP-35, pp. 373-383, 1987.

[14]. J. S. Lee, "Digital image enhancement and noise filtering by using local statistics," IEEE Trans. Pattern Anal. Machine Intell., vol. PAM1-2, 1980

[15]. "Refined filtering of image noise using local statistics," Comput. Graph. Image Process., vol. 15, pp. 380-389, 1981.

[16]. "Speckle suppression and analysis for synthetic aperture radar," Opt. Eng., vol. 25, no. 5, pp. 636-643, 1986.

[17]. A. Lopes, R. Touzi, and E. Nezry, "Adaptive speckle filters and scene heterogeneity," IEEE
Trans. Geosci. Remote Sensing, vol. 28, pp. 992-1000, 1990.

[18]. P. Perona and J. Malik, "Scale space and edge detection using anisotropic diffusion," IEEE Trans. Pattern Anal. Machine Intell., vol. 12, pp. 629-639, 1990.

[19]. D. Y. Tsai and S. Watanabe, "A method for optimization of fuzzy reasoning by genetic algorithms and its application to discrimination of myocardial heart disease," IEEE Trans. Nucl. Sci., vol. 46, pp. 2239-2246, Dec. 1999

[20]. J. Wang and X. Li, "A system for segmenting ultrasound images," in Proc. 14th Int. Conf. Pattern Recognition, vol. 1, 1998, pp. 456461.

[21]. S. H. Wong, K. L. Chan, and P. W. Fung, "Automati segmentation of ultrasonic images," in Proc. Computer, Communication, Control, Power Engineering (TENCON'93), vol. 2, IEEE Region 10 Conf., Part: 2, 1993, pp. 910913.

[22]. Mark A. Lubinski, Stanislav Y. Emelianov, and Matthew O’Donnell: "Speckle Tracking Methods for Ultrasonic Elasticity Imaging Using Short-Time Correlation" ieee transactions on ultrasonics, ferroelectrics, and frequency control, vol. 46, no. 1, january 1999.

[23]. Aleksandra $\mathrm{Pi}^{`}$ zurica, Wilfried Philips, Ignace Lemahieu, and Marc Acheroy "A Versatile Wavelet Domain Noise Filtration Technique for Medical Imaging" published in ieee transactions on medical imaging vol. 22, no. 3, march 2003, pages 323-331.

[24]. G.R.Arce and S.A.Fontana, "On the MidrangeEstimator," IEEE Trans. Acoust., Speech and Signal Processing, vol.ASSP-36, no.6, pp.920-922, June1988

[25]. A.C.Bovik, T.S.Huang and D.C.Munson, "A Generalisation of Median Filtering Using Linear Combinations of Order-Statistics," IEEE Trans. Acoust., Speech and Signal 
Processing, vol.ASSP-31, no.6, pp.1342-1349, Dec.1983.

[26]. I.Pitas and A.N. Venetsanopoulos, Nonlinear Digital Filters: Principles and Applications. Boston, MA:Kluwer Academic, 1990.

[27]. Y P Gowramma, Dr C N Ravikumar , "Development of novel fast block based trace mean correspondence algorithm for face tracking" International Conference Advanced computing and communications ,2006 Proceedings, IEEE pp 263- 266.

[28]. E.D SELEPCHI , O.G DULIU "Image processing and data analysis in computed tomography" PP665-675, Received September 12, 2006

\section{Cite this article as :}

Prof.(Dr.) Hitesh H Vandra, "Enhancement of Ultrasound Images (Removing of Speckle Noise Using Special An Isotropic Diffusion Filter)", International Journal of Scientific Research in Science, Engineering and Technology (IJSRSET), Online ISSN : 2394-4099, Print ISSN : 2395-1990, Volume 6 Issue 1, pp. 589-599, January-February 2019. Available at doi : https://doi.org/10.32628/IJSRSET1196191 Journal URL : https://ijsrset.com/IJSRSET1196191 